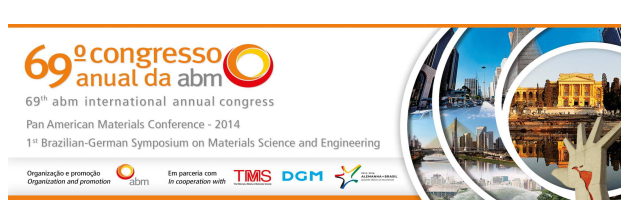

Tema: Materiais Cerâmicos, Compósitos e Poliméricos

\title{
COMPORTAMENTO MECÂNICO DE ESTRUTURAS COMPÓSITAS DE EPOXI COM NANOFIBRAS ELETROFIADAS DE NYLON 6*
}

\author{
João Paulo Ferreira Santos ${ }^{1}$ \\ Rosario Elida Suman Bretas ${ }^{2}$
}

\section{Resumo}

Neste trabalho, foram obtidas estruturas compósitas constituídas por camadas alternadas de epóxi com mantas de nanofibras de nylon 6. As nanofibras foram produzidas pela técnica de eletrofiação. O comportamento mecânico destas estruturas sob tração e sob impacto pendular IZOD foram estudados. A incorporação das nanofibras na matriz epoxídica permitiu aumentos no módulo de elasticidade $(6 \%)$, na resistência a tração $(7 \%)$ e na energia de impacto $(2 \%)$, ou seja, houveram ganhos de resistência mecânica sem sacrificar a resistência ao impacto, ao contrário do que ocorre na quase totalidade dos micro ou macromateriais modificadores do epóxi.

Palavras-chave: Epóxi; Eletrofiação; Nanofibras de nylon 6.

\section{MECHANICAL BEHAVIOR OF COMPOSITES STRUCTURES OF EPOXY/NYLON 6 ELECTROSPUN NANOFIBERS}

\section{Abstract}

In this work, composite structures consisting of alternating layers of epoxy and nylon 6 nanofibers mats were obtained. The nanofibers were produced by the electrospinning technique. The mechanical behavior of these structures under tensile and IZOD impact tests were studied. The incorporation of nanofibers allow the epoxy matrix increases the elastic modulus $(6 \%)$, the tensile strength $(7 \%)$ and the impact energy (2\%). Therefore, the gains in strength occurred without an impact resistance sacrifice. What does not occurs in almost all micro or macromodifiers materials of epoxy.

Keywords: Epoxy; Electrospinning; Nylon 6 nanofibers.

1 Engenharia de Materiais, mestre, Estudante de doutorado, Departamento de Engenharia de Materiais, UFSCar, São Carlos, SP, Brasil.

2 Engenharia Química, Doutor, Professor, Departamento de Engenharia de Materiais, UFSCar, São Carlos, SP, Brasil.

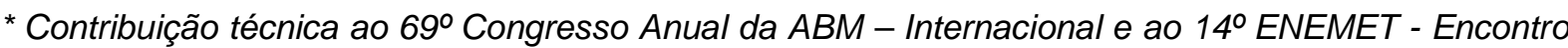
Nacional de Estudantes de Engenharia Metalúrgica, de Materiais e de Minas, 21 a 25 de julho de 2014, São Paulo, SP, Brasil.
} 


\section{INTRODUÇÃO}

Entre as diferentes classes de materiais compósitos, polímeros reforçados com fibras ou, do inglês: fiber reinforced polymer (FRP), são os mais comuns e os mais versáteis. Fibra e matriz polimérica são os dois constituintes base através dos quais uma arquitetura FRP específica pode ser concebida [1]. As propriedades físicomecânicas de tais compósitos podem ser ajustadas de forma eficiente por meio de uma seleção apropriada de ambos componentes e da fração relativa de cada um deles. Estes materiais conjugados vêm sendo fabricados comercialmente para vários segmentos da indústria, tais como em componentes aeroespaciais, automobilísticos e para fins militares [2].

As resinas epoxídicas são materiais termofixos estratégicos para fabricar FRPs: exibem elevada resistência mecânica, rigidez e dureza, baixa densidade, excelente resistência química e térmica, boa resistência à corrosão e baixa retração. [3]. No entanto, a grande desvantagem que inibe a sua propagação em várias aplicações industriais é a de que o epóxi curado é um material altamente reticulado e frágil com energias de fratura, em geral, duas ordens de grandeza inferior a dos termoplásticos de engenharia e três ordens menor que das ligas metálicas [4].

Em geral, o principal método para melhorar o comportamento frágil da resina epóxi consiste na incorporação de partículas de borracha em escala micrométrica. Geralmente, são utilizadas borrachas de copolímero butadieno-acrilonitrila terminadas com grupos carboxílicos (CTBNs). Porém, a adição de inclusões borrachosas dentro de uma matriz rígida resulta em uma redução significativa de resistência mecânica, tanto em termos do módulo de elasticidade quanto em termos de resistência à tensão. Em contraste, a utilização de partículas termoplásticas rígidas para tenacificar epóxis mostra-se como uma alternativa interessante para aumentar a energia de fratura destes materiais sem ocasionar perdas significativas em termos de resistência mecânica [5]. Por exemplo, Kim et al. [6] tenacificaram uma matriz epoxídica do diglicidil éter do bisfenol A (DGEBA) com nylon 6. Estes autores compararam 0 efeito de tenacificação da matriz epoxídica com micropartículas de nylon 6 e de CTBNs. Eles observaram que embora o efeito de tenacificação do nylon 6 tenha sido inferior aquele observado para o CTBN, praticamente não houveram perdas de módulo e de resistência à compressão, ao contrário do que aconteceu para o CTBN, para o qual perdas de até $50 \%$ de módulo foram observadas.

Neste contexto, a utilização de nanofibras termoplásticas como modificadores de matrizes poliméricas pode levar a propriedades novas e melhoradas em relação aos seus compostos homólogos em escala micro e macrométrica [7]. Nanocompósitos representam uma classe relativamente nova de materiais que apresentam fases em dimensões nanométricas, tipicamente entre 1 a $100 \mathrm{~nm}$. Como nanofibras apresentam elevada área superficial e elevada razão entre área e volume, isto é o fator chave para a melhoria da adesão interfacial que pode resultar em uma eficiente transferência de tensão da matriz polimérica para as nanofibras [8]. Além disso, quanto maior o diâmetro das fibras, maior será a possibilidade de se encontrar uma alta densidade de falhas ao longo da seção da fibra, o que é responsável pela redução da tensão de ruptura. Esta consideração é baseada num modelo que descreve as fibras como compostas de pequenos elementos unidos e a falha de todo o material ocorre quando qualquer destes elementos ou ligações falha [9]. Nanofibras fabricadas por eletrofiação são consideradas como promissores candidatos para a modificação em compósitos. Elas são continuas fibras longas, que

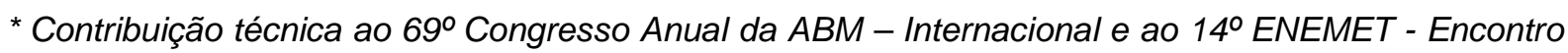
Nacional de Estudantes de Engenharia Metalúrgica, de Materiais e de Minas, 21 a 25 de julho de 2014, São Paulo, SP, Brasil. 
diferentemente de outras nanofibras com comprimentos menores produzidas por outras técnicas mostram uma excelente razão de aspecto, já que apresentam comprimentos da ordem de centímetros e diâmetros da ordem de nanômetros. Apresentam um número significativamente menor de bordas que atuam como pontos concentradores de tensão [10].

Os nylons são termoplásticos de engenharia com excelentes propriedades mecânicas. Inúmeros trabalhos obtiveram nanofibras de nylon 6 através da técnica de eletrofiação [11-13]. Nanofibras eletrofiadas de nylon 6 foram empregadas com sucesso como modificadores de algumas matrizes poliméricas. Por exemplo, RomoUribe et al. [14] utilizaram nanofibras de nylon 6 como reforço em filmes condutores de Polianilina, um polímero tipicamente condutor. A análise dinâmico mecânica revelou um grande aumento de módulo de armazenamento nos compósitos a temperatura ambiente, que possibilitou o aumento da temperatura de trabalho dos filmes de Polianilina de 100 para $200^{\circ} \mathrm{C}$. Chen et al. [15] desenvolveram compósitos de Poli(metil metacrilato) (PMMA) reforçados com nanofibras eletrofiadas híbridas de nylon 6 com PMMA. Estes autores realizaram uma co-eletrofiação, com o nylon 6 formando o núcleo da fibra e o PMMA como a "casca". Dez camadas de mantas destas nanofibras foram empilhadas de forma alternada com PMMA na forma de pó, este arranjo foi disposto em uma prensa a quente a $185^{\circ} \mathrm{C}$. Este tratamento fundiu o PMMA, inclusive aquele da casca das nanofibras, enquanto que restaram as nanofibras de nylon 6 corresponde ao núcleo das pré-fibras híbridas. O resultado foi um compósito transparente, que mostrou um aumento de $38 \%$ de módulo em relação ao PMMA puro enquanto que uma redução muito pequena de transparência óptica foi observada. Utilizando o mesmo método por moldagem por compressão, Neppali et al. [16] fabricaram compósitos de Policaprolactona com nanofibras de nylon 6. Os compósitos apresentaram aumentos de $32 \%$ em módulo e $24 \%$ na ductilidade, diferentemente do que é observado em compósitos contendo outros tipos de cargas, onde o aumento de módulo acontece as custas de uma queda na elongação. Neste trabalho, nós propusemos a fabricação por moldagem por compressão de estruturas de nanocompósitos constituídas por camadas alternadas de epóxi e nanofibras de nylon 6 para avaliar o efeito das nanofibras nas propriedades mecânicas do epóxi sob tração e sob resistência ao impacto IZOD.

\section{MATERIAIS E MÉTODOS}

\subsection{Materiais}

Nylon6 (resina de especificação Mazmid B400, fornecida pela Mazzaferro Indústria e Comércio de Polímeros e Fibras Ltda) na forma de pellets. Ácido fórmico adquirido da PA Synth; Sistema de resina termofixa epóxi DGEBA (HY 364) e endurecedor Trietileno tetramida (TETA), fornecidos pela Huntsman.

\subsection{Métodos}

\subsubsection{Eletrofiação e produção das nanofibras}

Uma solução de nylon 6 em ácido fórmico de concentração $0,18 \mathrm{~g} / \mathrm{ml}$ foi preparada a temperatura ambiente sob intensa agitação magnética. A mistura resultante foi eletrofiada em um equipamento e montagem já descrito num trabalho anterior [12] que consiste de uma seringa, fonte de alta tensão e coletor rotativo. A seringa tem uma agulha de aço de $0,5 \mathrm{~mm}$ de diâmetro; a distância entre a ponta da agulha e 0

\footnotetext{
* Contribuição técnica ao 69 Congresso Anual da ABM - Internacional e ao 14ํㅡㄹ ENEMET - Encontro Nacional de Estudantes de Engenharia Metalúrgica, de Materiais e de Minas, 21 a 25 de julho de 2014, São Paulo, SP, Brasil.
} 


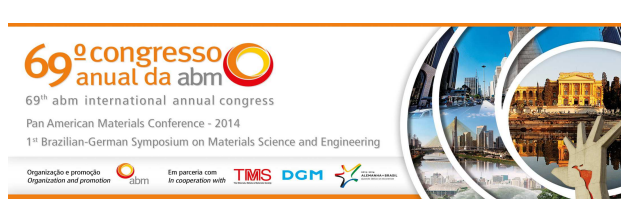

coletor foi ajustada em $7 \mathrm{~cm}$; a diferença de potencial aplicada entre a ponta da agulha e o coletor foi de $30 \mathrm{kV}$; a velocidade de rotação empregada para o coletor para a deposição das nanofibras foi de 2000rpm. A temperatura e a umidade do ambiente de eletrofiação ficaram entre $23-25^{\circ} \mathrm{C}$ e $40-50 \%$, respectivamente.

\subsubsection{Estruturas de nanocompósitos}

A resina DGEBA foi misturada com o endurecedor TETA na razão TETA/DGEBA de $1: 10(\mathrm{~m} / \mathrm{m})$ e em seguida foi impregnada de forma alternada com as mantas tratadas para formar as estruturas como esquematizado na figura 1 abaixo, na forma de sanduiches. Foram produzidos estruturas com quatro concentrações em volume de nanofibras de nylon 6: 0, 3, 7 e 15\% (v/v). A cura foi realizada a $60^{\circ} \mathrm{C}$ por 6 horas sob vácuo e pressão de $5 \mathrm{kPa}$ em uma prensa Marconi modelo MA098 aquecida por resistências.

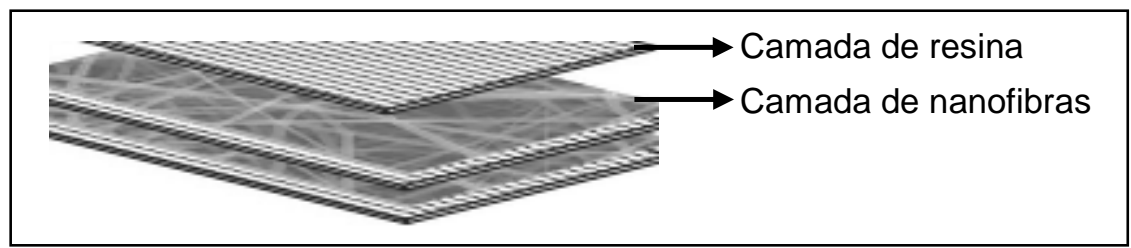

Figura 1. llustração esquemática de uma estrutura compósita constituída por camadas alternadas de resina epoxídica e mantas de nanofibras eletrofiadas.

\subsubsection{Caracterização}

As mantas obtidas na eletrofiação e as estruturas de nanocompósitos foram caracterizadas por microscopia eletrônica de varredura (MEV). O objetivo, no caso das mantas, foi o de avaliar a morfologia das nanofibras, grau de integridade e presença de defeitos. A distribuição estatística de diâmetros das nanofibras foi medida e calculada com auxílio do programa IMAGE PRO PLUS 4.5. No caso das estruturas de nanocompósitos, o objetivo foi o de avaliar se o sistema final de fato se constituía num nanocompósito com nanofibras em meio a matriz epoxídica, bem como avaliar o grau de integridade das nanofibras após a incorporação. Foi utilizado um microscópio modelo FEG XL 30 da Philips operando entre 15 e 20kV.

Os testes de tração foram realizados em uma maquina de ensaios Instron, modelo 5569 seguindo a norma ASTM D-638 a uma taxa de carregamento de $5 \mathrm{~mm} / \mathrm{min}$. Os testes de resistência ao impacto IZOD foram realizados em uma maquina de ensaios CEAST, utilizando um martelo de $1 \mathrm{~J}$, seguindo a norma ASTM D-256; os entalhes das amostras foram feitos com uma profundidade de $2,5 \mathrm{~mm}$ nesta mesma maquina.

\section{RESULTADOS E DISCUSSÃO}

A micrografia da figura 2(A) mostra as nanofibras obtidas por eletrofiação e a figura 2(B) a distribuição estatística de diâmetros. As mantas consistem de nanofibras entrelaçadas que se depositaram sobre tiras de papel alumínio dispostas sobre o coletor rotativo. As fibras exibem poucos defeitos e muitos vazios entre elas, característica típica de nanofibras eletrofiadas. Foi calculado um diâmetro médio de $100,3 \mathrm{~nm}$ para a amostra representada na figura $2(\mathrm{~A})$.

\footnotetext{
* Contribuição técnica ao $69^{\circ}$ Congresso Anual da ABM - Internacional e ao 14ํㅡㄹ ENEMET - Encontro Nacional de Estudantes de Engenharia Metalúrgica, de Materiais e de Minas, 21 a 25 de julho de 2014, São Paulo, SP, Brasil.
} 

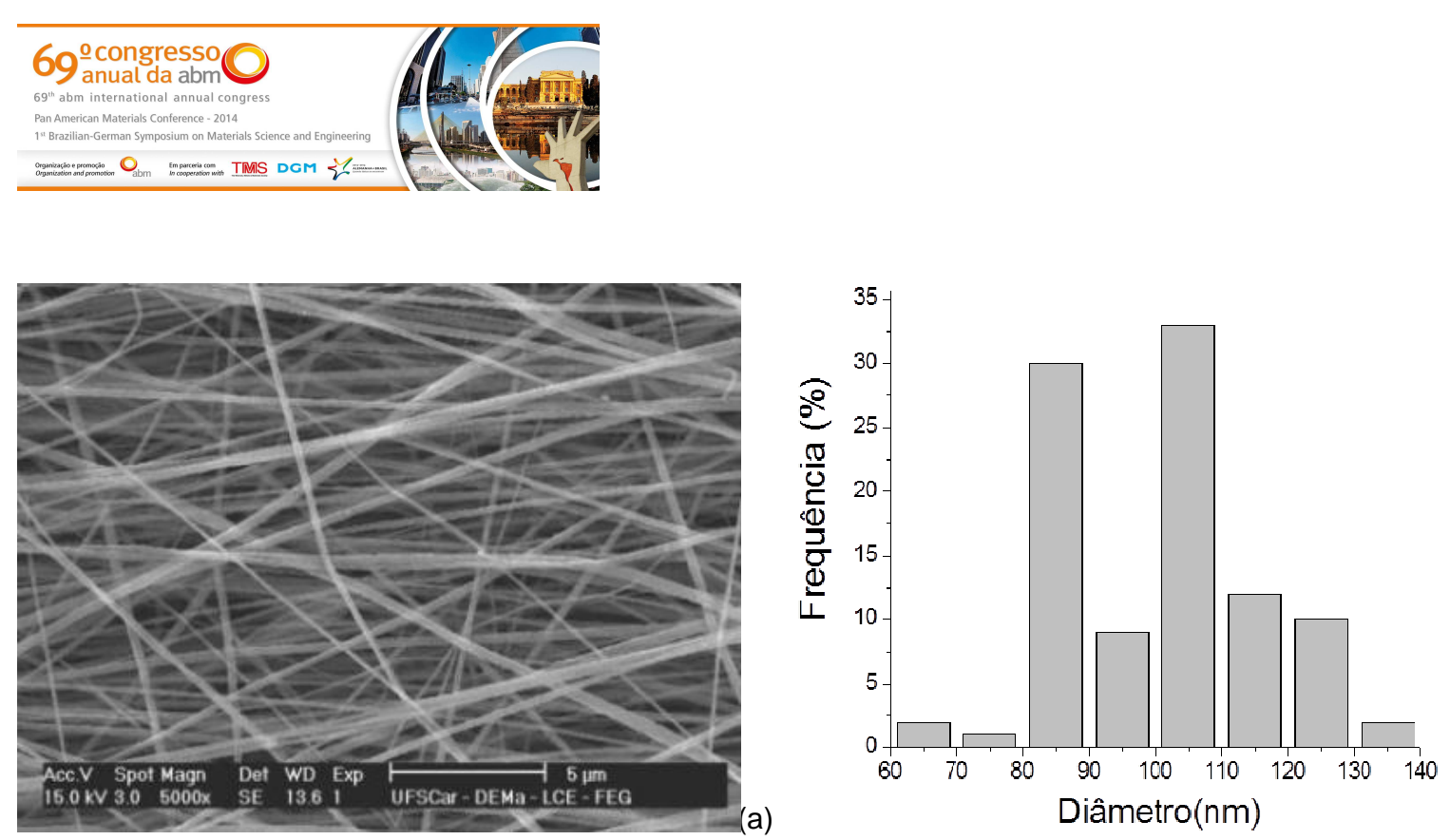

Figure 2. (A) Micrografia de MEV com aumento de 5000x e (B) distribuição estatística de diâmetros para as mantas eletrofiadas.

As figuras $3(A)$ e $3(B)$ mostram figuras dos corpos de prova utilizados para os testes mecânicos de tração e de impacto, respectivamente. Pode-se notar que os corpos de prova constituídos por nanofibras de nylon praticamente não diferem de cor entre si e em relação ao epóxi puro. Todos apresentaram um aspecto translucido róseo. As nanofibras quase não podem ser vistas no interior destas estruturas. Este resultado corrobora com resultados observados por outros autores [16], que atribuíram isto ao fato das nanofibras eletrofiadas apresentam diâmetros da ordem de grandeza dos comprimentos de onda da luz visível.
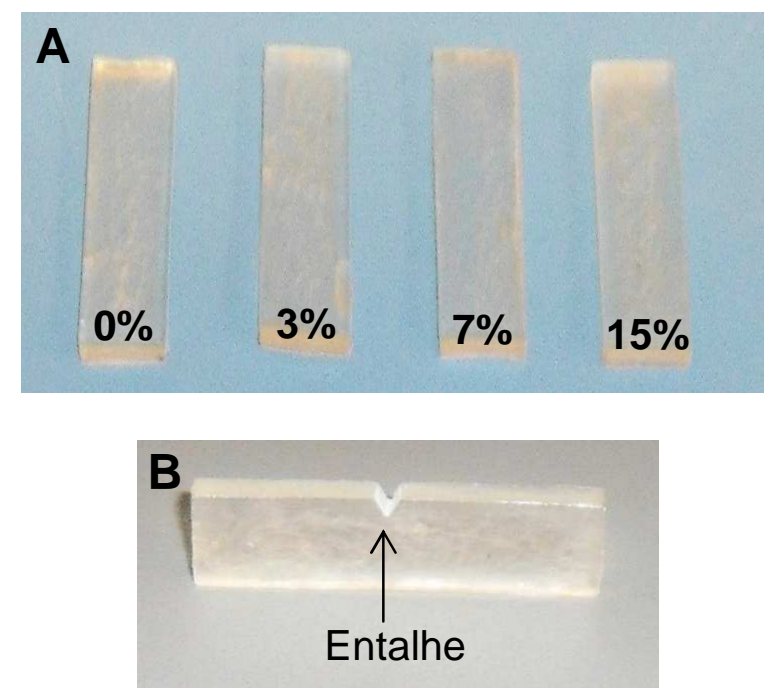

Figura 3. (A) Corpos de prova para ensaio de tração e (B) Corpos de prova para ensaio de resistência ao impacto IZOD.

A figura 4 mostra as curvas de tensão em função da deformação obtidas através dos ensaios de tração uniaxial e a tabela 1 mostra os valores de módulo elástico $(E)$, de tensão na ruptura $\left(\sigma_{r}\right)$, de deformação na ruptura $\left(\varepsilon_{r}\right)$, de tenacidade (área sob as curvas) e, na ultima coluna os valores de energia de impacto medidos no ensaio de resistência ao impacto IZOD. Analisando-se os dados pôde-se identificar que a adição de uma fração de $15 \%$ (v/v) de nanofibras resultou em um aumento de $6 \%$ no módulo e de $7 \%$ na resistência à ruptura. Embora estes aumentos sejam baixos se comparados ao reforço proporcionado por fibras de alto módulo como as de vidro ou

\footnotetext{
* Contribuição técnica ao $69^{\circ}$ Congresso Anual da ABM - Internacional e ao 14ํㅡㄹ ENEMET - Encontro Nacional de Estudantes de Engenharia Metalúrgica, de Materiais e de Minas, 21 a 25 de julho de 2014, São Paulo, SP, Brasil.
} 
de carbono [2], a deformação na ruptura e a tenacidade ficaram praticamente constantes e não caíram com o aumento da fração de nanocarga. Os resultados obtidos a partir do ensaio de resistência ao impacto IZOD mostraram que a adição de $15 \%(\mathrm{v} / \mathrm{v})$ de mantas de nanofibras resultou num aumento de $2 \%$ na resistência ao impacto das estruturas, isto é, não a piorou. Embora não tenha havido um grande aumento na resistência ao impacto, os resultados mostraram-se bastante promissores uma vez que um leve aumento no modulo de elasticidade foi conseguido sem sacrificar a ductilidade e a resistência ao impacto das estruturas.

Tabela 1. Valores de módulo elástico $(E)$, tensão de ruptura $\left(\sigma_{r}\right)$, deformação de ruptura $\left(\varepsilon_{r}\right)$, tenacidade e energia de impacto IZOD (EI) para as amostras.

\begin{tabular}{cccccc}
\hline Sample & $\mathrm{E}(\mathrm{GPa})$ & $\sigma_{\mathrm{r}}(\mathrm{MPa})$ & $\varepsilon_{\mathrm{r}}(\%)$ & $\begin{array}{c}\text { Tenacidade } \\
\left(\mathrm{MJ} / \mathrm{m}^{3}\right)\end{array}$ & $\mathrm{El}(\mathrm{J} / \mathrm{m})$ \\
\hline Puro & $1,9 \pm 0,0$ & $22,1 \pm 1,8$ & $4,2 \pm 0,7$ & $0,81 \pm 0,02$ & $12,86 \pm 0,2$ \\
$3 \%$ & $1,9 \pm 0,0$ & $23,4 \pm 3,2$ & $4,4 \pm 0,4$ & $0,86 \pm 0,01$ & $12,89 \pm 0,2$ \\
$7 \%$ & $2,0 \pm 0,0$ & $24,0 \pm 2,1$ & $4,4 \pm 0,3$ & $0,88 \pm 0,02$ & $12,94 \pm 0,1$ \\
$15 \%$ & $2,1 \pm 0,0$ & $25,1 \pm 2,7$ & $4,2 \pm 0,3$ & $0,87 \pm 0,01$ & $13,07 \pm 0,1$ \\
\hline
\end{tabular}

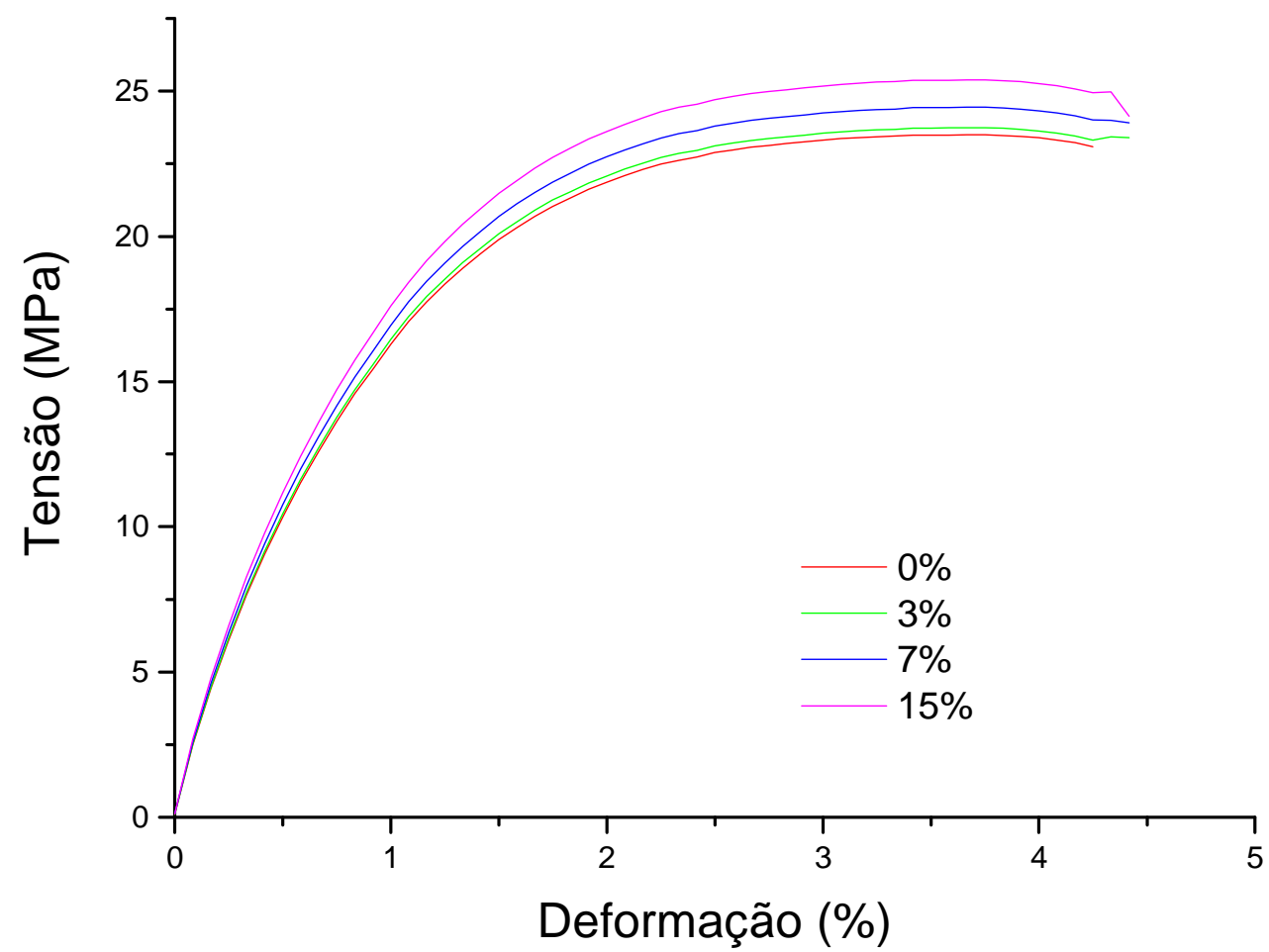

Figura 4. Curvas da tensão em função da deformação para estruturas obtidas.

A figura 5 abaixo mostra uma micrografia de MEV da seção de fratura da estrutura compósita com $0,15 \%(\mathrm{v} / \mathrm{v})$ de nanofibras. Através da micrografia foi possível observar que as mantas de nanofibras foram adequadamente incorporadas em meio

\footnotetext{
* Contribuição técnica ao 69ำ Congresso Anual da ABM - Internacional e ao 14ํㅡㄹ ENEMET - Encontro Nacional de Estudantes de Engenharia Metalúrgica, de Materiais e de Minas, 21 a 25 de julho de 2014, São Paulo, SP, Brasil.
} 


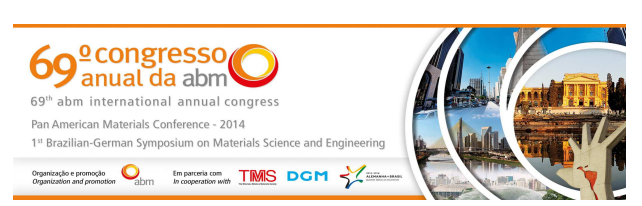

a matriz epoxídica e as nanofibras se mantiveram integras nestas estruturas mesmo após a incorporação.

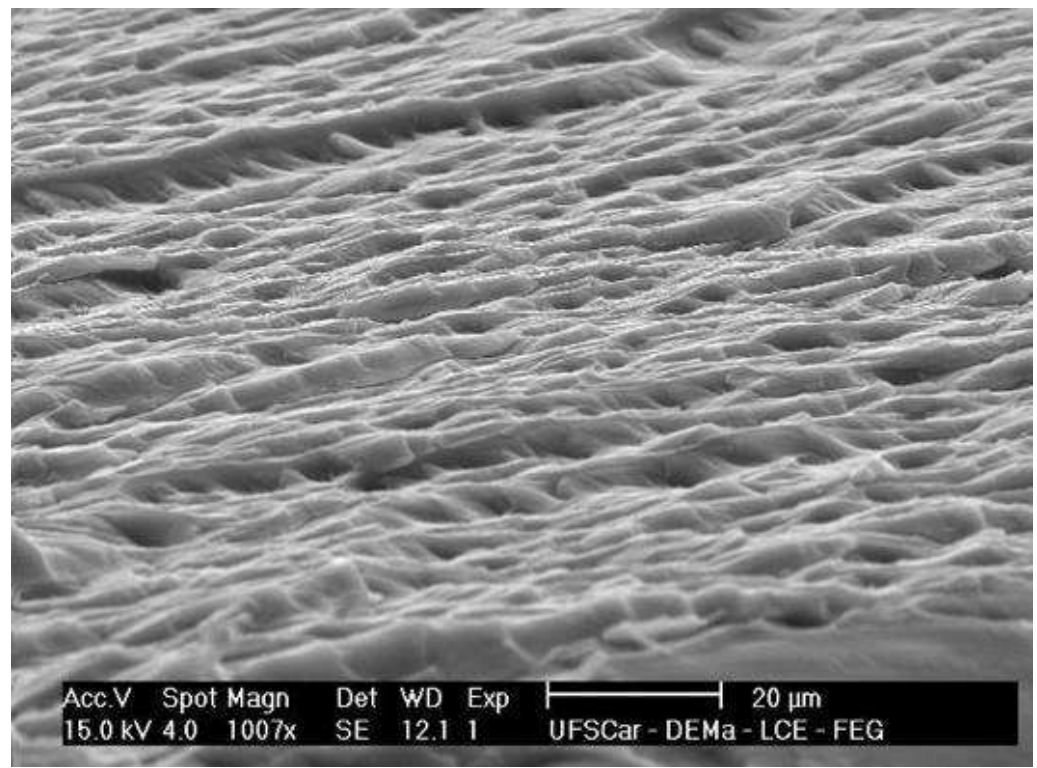

Figura 5. Micrografia de MEV de amostra de estrutura compósita criofraturada. A amostra é composta por $15 \%(\mathrm{v} / \mathrm{v})$ de mantas da PA6. Aumento de 1000x.

\section{CONCLUSÃO}

Estruturas de nanocompósitos de epóxi com mantas de nanofibras eletrofiadas de nylon 6 foram obtidas. Através da técnica de eletrofiação foram obtidas nanofibras com poucos defeitos. A incorporação de nanofibras de nylon 6 na matriz epoxídica resultou em um leve aumento no módulo, na resistência à ruptura e na energia de impacto IZOD. Ainda que os aumentos nestas três propriedades tenham sido pequenos, a utilização destes nanomateriais como modificadores da matriz epoxídica permitiram que as três propriedades fossem aumentadas, ou seja, que houvesse um ganho de resistência mecânica sem sacrifício da resistência ao impacto, ao contrário do que ocorre na quase totalidade dos micro ou macromateriais modificadores do epóxi.

\section{Agradecimentos}

Os autores agradecem a FAPESP e ao CNPQ pelo auxilio financeiro.

\section{REFERÊNCIAS}

1 Nielsen LE. Mechanical properties of polymers and composites, Wiley, $2^{\underline{a}}$ edição, 1998

2 Callister WD. Ciência e Engenharia de Materiais: Uma Introdução, 5ae edição. LCT, 2000.

3 Lee H, Neville K. Handbook of Expoy Resin, McGraw Hill, New York, 1947.

4 Bascom WD, Hunston DL. In Rubber Toughened Plastics, American Chemical Society, Washington, Advances in Chemistry Series, 1989

5 Huang Y, Hunston DI, Kinloch A J, Riew C, K. Mechanisms of Toughening Thermoset Resins. Toughened Plastics, 1993; 1: 1-38,

6 Kim JK, Robertson RE. Toughening of thermoset polymers by rigid crystalline particles. Journal of Materials Science, 1992; 27:161-174.

\footnotetext{
* Contribuição técnica ao $69^{\circ}$ Congresso Anual da ABM - Internacional e ao 14ํㅡㄹ ENEMET - Encontro Nacional de Estudantes de Engenharia Metalúrgica, de Materiais e de Minas, 21 a 25 de julho de 2014, São Paulo, SP, Brasil.
} 
